\title{
Transmission mode switching for device-to-device communication aided by relay node
}

\author{
Yiyang $\mathrm{Ni}^{1}$, Dan Qiao ${ }^{2}$, Xiao $\mathrm{Li}^{2}$, Shi $\mathrm{Jin}^{2}$ and Hongbo Zhu ${ }^{1 *}$
}

\begin{abstract}
In this paper, we investigate the strategy of transmission mode switching for device-to-device (D2D) communication in both single-cell scenario and multi-cell scenarios, which selects the transmission mode to guarantee the maximum ergodic achievable sum-rate among three transmission modes. We first introduce the basic operation principles of three communication transmission modes which are named as traditional cellular communication mode, direct D2D communication mode and two-way decode-and-forward (DF)-relayed D2D communication mode. Then we derive the corresponding expressions for the ergodic achievable sum-rates of each transmission mode, and get the crossing points of different transmission modes to attain maximum ergodic achievable sum-rate of the system. From the analytical results, we can see that the proper operating region of each transmission mode is related to different interference level and distance of the D2D users. Based on the analytical results, we obtain a reliable communication transmission mode switching strategy which guarantees the system to choose the mode with the maximum ergodic achievable sum-rate so as to improve the performance of D2D communication. Numerical results demonstrate that by applying mode switching, the ergodic achievable sum-rate of the system achieves a remarkable enhancement.
\end{abstract}

Keywords: D2D communication; Two-way relay; Transmission mode switching; Ergodic achievable sum-rate

\section{Introduction}

With the rapid increase of context-aware applications, online game and various innovative multi-media services, more spectral efficient communication techniques are required for future wireless networks [1]. Recently, deviceto-device (D2D) communication underlying cellular networks has attracted considerable attention due to its much improved spectral efficiency [2,3]. D2D communications commonly refer to the technologies that enable devices to communicate directly without an infrastructure of access points or base stations [4,5]. Despite the advantages D2D communication has, the inter-channel interference (ICI) between cellular and D2D links challenges the entire system performance, since the D2D link reuses the cellular frequency resource [6,7]. Several methods have been proposed to solve this problem, including transmission power

\footnotetext{
${ }^{*}$ Correspondence: zhuhb@njupt.edu.cn

1 Jiangsu Key Laboratory of Wireless Communications College of

Telecommunications and Information Engineering, Nanjing University of Posts and Telecommunications, Nanjing 210003, China

Full list of author information is available at the end of the article
}

control of D2D users [8] or cellular users [9], orthogonal or non-orthogonal resource allocation schemes $[10,11]$, and so on.

Meanwhile, relay-assisted communication has attracted lots of interests due to its great potential in enhancing the system performance [12]. Spectral efficient protocols for relay channels have been proposed including the two-way amplify-and-forward (AF), decode-and-forward (DF) and compress-and-forward (CF) relay protocols in $[13,14]$. The ergodic achievable rates have been studied for DF relaying system in $[15,16]$. A recent study [17] discussed the outage probability of the D2D communication aided by a two-way DF relay node for both the asymmetric and symmetric cases. The result shows that the new strategy gains advantages in outage probability over that of the traditional strategy without extra power. The authors in [18] proposed a joint precoded-and-decoder scheme in D2D communication system to mitigate interference and improve the error performance. In [19], the authors presented two coding-based relaying schemes for D2D communication system which can exploit the

\section{是 Springer}


transmission opportunities for the D2D users. The outage behavior was investigated for the multi-hop one-way decode-and-forward relay-assisted D2D communication in [20]. The performance of achievable capacity for the relay-assisted D2D communication was studied in [21]. Wang [22] derived out the interference constrained criteria of employing the D2D communication with relayassisted mode based on the outage performance. A relay selection rule was deduced on the basis of constraining the interference in the permitted range which considers the achievable rate [23].

The communication transmission mode switching has always been an issue of great contention in wireless communication study [24-26]. A distributed mode selection algorithm switching between TDMA and SDMA was proposed in [24], where each user feeds back its preferred mode and the channel quality information. In [25], an SU/MU mode switching algorithm was proposed for the ZF precoding system considering delayed and quantized CSIT. The mode switching point can be explicitly derived based on the parameters including average SNR, normalized Doppler frequency, and codebook size, which are computable at base station (BS). In [26], the authors pointed out that different receiving modes can be exploited at $\mathrm{D} 2 \mathrm{D}$ receivers according to the interference level, and proposed a new method of exploiting a retransmission of the interference from the BS for middle interference level region.

In this paper, we not only consider the traditional cellular communication mode and the direct D2D communication mode, but also take the relay aided strategy into consideration, which provide a flexible and effective transmission mode switching strategy in D2D communication underlying cellular networks. We investigate the basic operation principles of different transmission modes, and derive accurate approximations for ergodic achievable sum-rates for both single-cell and multi-cell scenarios. A transmission mode switching strategy, which select the one with the highest ergodic achievable sumrate among all transmission modes, is proposed based on the ergodic sum-rate as a function of the interference from cellular users and the distance between D2D users.

The rest of this paper is organized as follows: We first introduce three transmission modes of D2D communication for both single-cell and multi-cell scenarios in Section 2. The basic operation principle of the three transmission modes along with the expressions of their ergodic achievable sum-rates are derived in closed forms respectively in Section 3. According to the crossing points of different transmission modes, the mechanism for transmission mode switching which selects the transmission mode guaranteeing the maximum ergodic achievable sum-rate is explained in Section 4. In Section 5, numerical results are provided to confirm the analytical results, showing the reliability of the transmission mode switching strategy. Finally, we conclude the paper in Section 6.

\section{System model}

In this paper, we consider the D2D communication underlaying cellular network in the single-cell scenario and multi-cell scenario, respectively, as illustrated in Figures 1 and 2. The single-cell scenario involves one cellular user (i.e., UEC) and a pair of D2D users (i.e., UE1, UE2) and we only take into account the intra-cell interference caused by
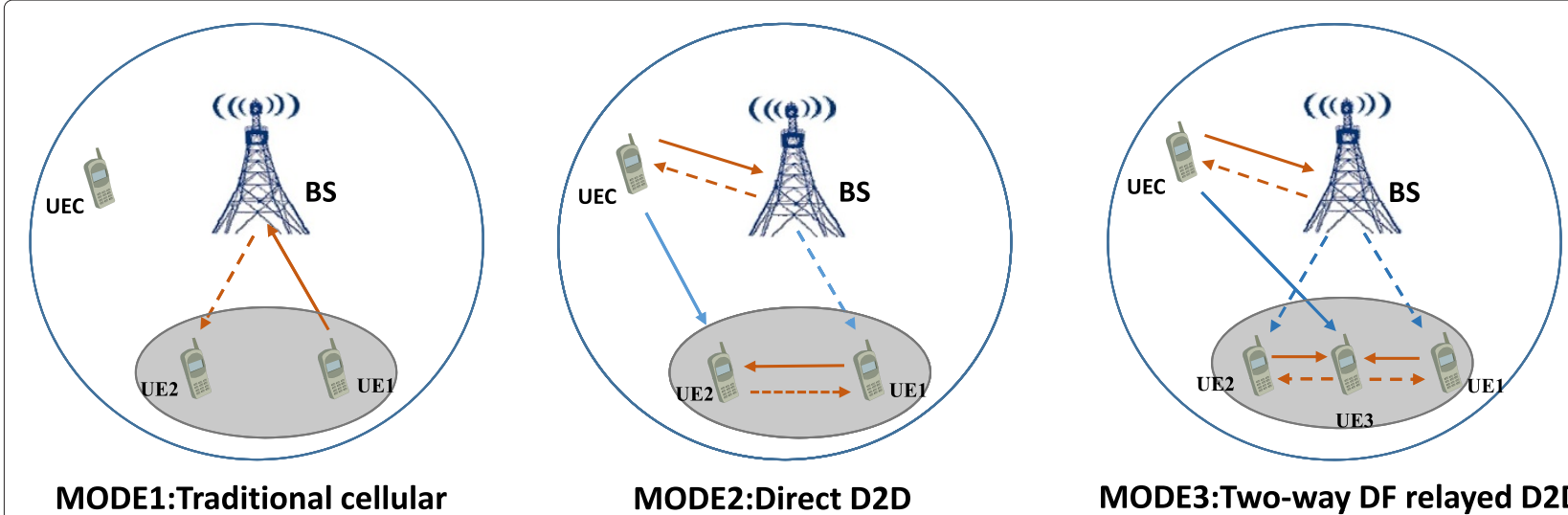

MODE3:Two-way DF relayed D2D

$\longrightarrow$ First time slot, desired signal

$----\rightarrow$ Second time slot, desired signal

First time slot, interference signal

$---\rightarrow$ Second time slot, interference signal

Figure 1 Schematic diagram of D2D communication system in a single-cell scenario where three transmission modes are provided. 


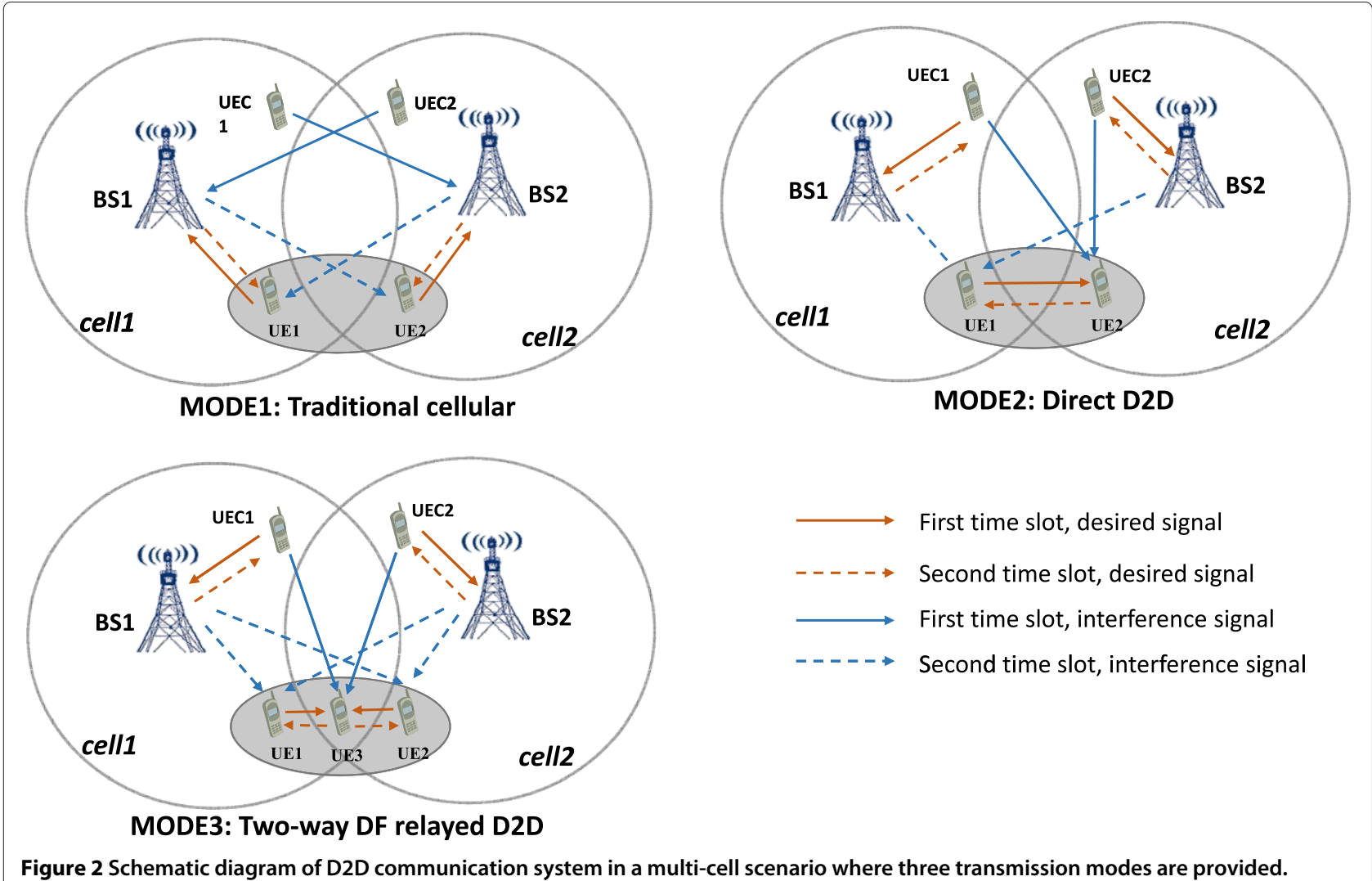

the coexistence of a cellular user and a D2D pair. For the multi-cell scenario, we consider a cellular system with two cells for simplification, but the results can be extended to scenarios with more than two cells. As shown in Figure 2, in each cell, the BS controls a cellular user (UEC1 in cell1 and UEC2 in cell2) and a D2D user (UE1 in cell1 and UE2 in cell2). Relay node UE3 lays between the D2D users. While all cellular users communicate directly with the BS, D2D users can either exploit the direct link or communicate via the BS.

We assume that the BS in each cell and all the users are equipped with a single antenna. The uplink and downlink channels are assumed to be reciprocal and invariant, and are based on two consecutive equal time slots. UE1 and UE2 are in close proximity to one another. There are three transmission modes in this communication system including traditional cellular communication mode (referred to as MODE1), direct D2D communication mode (MODE2), and two-way DF-relayed D2D communication mode (MODE3).

We denote the transmission power of terminal $i$ as $P_{i}(i=B, C, D, 1,2,3)$, where $B$ represents the BS, $C$ refers to the cellular user, $D$ stands for the D2D users and other integers denote the corresponding UE terminals. It is assumed that the transmit power of the base stations are the same while the transmit power of each user is equal. For fading channels, we assume that the channel coefficients between terminal $i$ and terminal $j$ $(j=B, C, D, 1,2,3), h_{i j} \sim C N(0,1)$, are statistically independent across users and satisfy $h_{i j}=h_{j i}$. We use $n_{i}$ to indicate zero-mean complex Gaussian noise with variance of $N_{0}$. According to [17], the received power $P_{i j}$ at user $j$ measured at $d_{i j}$ away from the transmitter user $i$ is

$$
P_{i j}=P_{i} d_{i j}^{-\alpha}
$$

where $\alpha$ is the pass-loss exponent. For the convenience of analysis, we denote $\beta=d_{12} / 2 d_{\mathrm{BD}}$ and $g=d_{\mathrm{BD}}^{-\alpha}$. Throughout this paper, the superscripts $(\cdot)^{s}$ and $(\cdot)^{m}$ represent the single-cell and the multi-cell scenarios, respectively. For example, $R_{\text {sum,tra }}^{s}$ stands for the ergodic achievable sum-rate of the traditional cellular communication mode (MODE1) in the single-cell scenario while $R_{\text {sum,tra }}^{m}$ stands for the ergodic achievable sum-rate of MODE1 in the multi-cell scenario.

\section{Ergodic achievable sum-rates}

In this section, we first introduce three communication transmission modes including: traditional cellular communication mode, direct D2D communication mode and two-way DF relayed D2D communication mode. Based on the definition of ergodic achievable sum-rate, we then 
derive the corresponding expressions for these transmission modes in both single-cell and multi-cell scenarios.

\subsection{MODE1: traditional cellular communication mode}

For the single-cell scenario, when the D2D users communicate with each other through the traditional cellular communication mode, the cellular users do not generate any interference to them because each link is separated by different frequency resources. But for the multi-cell scenario, both the BS and D2D users receive interference from the other cell. Therefore, we discuss the ergodic achievable sum-rate of this mode in the single-cell and multi-cell scenarios, respectively.

\subsubsection{Single-cell scenario}

As shown in Figure 1, in MODE1 when the D2D users want to communicate with each other, UE1 sends the desired message to the BS in the first time slot and the BS sends it to UE2 in the next time slot. This procedure is free of interference from the cellular user. The received signalto-interference-plus-noise ratio (SINR) for the UE1-BS link and the BS-UE2 link can be respectively obtained as follows:

$$
\gamma_{i}^{s}=\frac{P_{D} g\left|h_{\mathrm{Bi}}\right|^{2}}{N_{0}},(i=1,2) .
$$

Then the ergodic achievable sum-rate of this transmission mode is given by

$$
R_{\text {sum,tra }}^{S}=\min \left\{R_{1 B, \text { tra }}^{S}, R_{B 2, \text { tra }}^{S}\right\},
$$

where

$$
R_{1 B, \text { tra }}^{s}=\frac{1}{2} E\left\{\log \left(1+\frac{P_{D}}{g}\left|h_{1 B}\right|^{2} N_{0}\right)\right\}
$$

and

$$
R_{B 2 \text {,tra }}^{S}=\frac{1}{2} E\left\{\log \left(1+\frac{P_{B}}{g}\left|h_{B 2}\right|^{2} N_{0}\right)\right\} .
$$

Based on Jensen's inequality

$$
E\{\log (1+x)\} \leq \log (1+E\{x\}),
$$

we can simplify the expression of ergodic achievable sumrate as

$$
R_{\text {sum,tra }}^{s} \approx \frac{1}{2} \min \left\{\log \left(1+\frac{P_{D} g}{N_{0}}\right), \log \left(1+\frac{P_{B} g}{N_{0}}\right)\right\} .
$$

It is known that $P_{D}<P_{B}$, thus the ergodic achievable sum-rate of MODE1 can be approximated as

$$
R_{\text {sum,tra }}^{S}=\frac{1}{2} \log \left(1+\frac{P_{D} g}{N_{0}}\right) .
$$

\subsubsection{Multi-cell scenario}

For the multi-cell scenario, as illustrated in Figure 2, UE1 sends the desired messages to the BS in cell1 while UE2 sends message to the BS in cell 2 during the first time slot. However, the cellular user produces the interference with each other, which is different from the single-cell scenario that no interference exists. Therefore, the received SINR for each link can be obtained as

$$
\gamma_{i B_{i}}^{m}=\frac{P_{D} g\left|h_{i B_{i}}\right|^{2}}{P_{D} d_{j B_{i}}^{-\alpha}\left|h_{j B_{i}}\right|^{2}+N_{0}},(i, j=1,2 ; i \neq j) .
$$

In the second time slot, BS1 sends the received message from UE2 to UE1 while BS2 sends the message from UE1 to UE2 to complete the communication process. During this period, the BSs contribute the interference to the users in the other cell, and the received SINR of BS1-UE1 and BS2-UE2 link is given by

$$
\gamma_{B_{i} i}^{m}=\frac{P_{B}}{g}\left|h_{B_{i} i}\right|^{2} P_{B} d_{B_{j} i}^{-\alpha}\left|h_{B_{j} i}\right|^{2}+N_{0},(i, j=1,2 ; i \neq j) .
$$

Based on (9) and (10), the ergodic achievable sum-rate of traditional cellular communication mode in a multi-cell scenario is given by

$$
R_{\mathrm{sum}, \mathrm{tra}}^{m}=R_{1, \mathrm{tra}}^{m}+R_{2, \text { tra }}^{m}
$$

where

$$
R_{1, \text { tra }}^{m}=\frac{1}{2} \min \left\{E\left\{\log \left(1+\gamma_{1 B_{1}}^{m}\right)\right\}, E\left\{\log \left(1+\gamma_{B_{2} 2}^{m}\right)\right\}\right\}
$$

and

$$
R_{2, \text { tra }}^{m}=\frac{1}{2} \min \left\{E\left\{\log \left(1+\gamma_{2 B_{2}}^{m}\right)\right\}, E\left\{\log \left(1+\gamma_{B_{1} 1}^{m}\right)\right\}\right\} .
$$

In order to compare the performance of each mode, the more concise results are needed. Having the following inequations

$$
E\left\{\log \left(1+\frac{z}{y+N_{0}}\right)\right\} \leq \log \left(1+E\left\{\frac{z}{y+N_{0}}\right\}\right)
$$

and

$$
E\left\{\frac{z}{y+N_{0}}\right\} \approx \frac{E\{z\}}{E\{y\}+N_{0}},
$$

we then get the approximations of (12) and (13) by using the inequations in (14) and (15)

$$
\begin{aligned}
R_{1, \text { tra }}^{m} \approx \frac{1}{2} \min & \left\{\log \left(1+\frac{P_{D} g}{P_{D} d_{2 B_{1}}^{-\alpha}+N_{0}}\right),\right. \\
& \left.\times \log \left(1+\frac{P_{B} g}{P_{B} d_{B_{1} 2}^{-\alpha}+N_{0}}\right)\right\}
\end{aligned}
$$


and

$$
\begin{aligned}
R_{2, \text { tra }}^{m} \approx \frac{1}{2} \min & \left\{\log \left(1+\frac{P_{D} g}{P_{D} d_{1 B_{2}}^{-\alpha}+N_{0}}\right)\right. \\
& \left.\times \log \left(1+\frac{P_{B} g}{P_{B} d_{B_{2}}^{-\alpha}+N_{0}}\right)\right\} .
\end{aligned}
$$

As $P_{D}<P_{B}$, the ergodic achievable sum-rate of MODE1 in a multi-cell scenario is presented as

$$
\begin{aligned}
R_{\text {sum,tra }}^{m}= & \frac{1}{2} \log \left(1+\frac{P_{D} g}{P_{D} d_{2 B_{1}}^{-\alpha}+N_{0}}\right) \\
& +\frac{1}{2} \log \left(1+\frac{P_{D} g}{P_{D} d_{1 B_{2}}^{-\alpha}+N_{0}}\right) .
\end{aligned}
$$

\subsection{MODE2: direct D2D communication mode}

For the direct D2D communication mode, the interference scenarios of single-cell and multi-cell are described in MODE2 of Figures 1 and 2, where cellular users transmit messages to the base stations while UE1 sends messages to UE2. As reusing the same time-frequency resources in the first time phase, D2D and cellular users cause interferences to each other. The interference from D2D users to cellular users can be managed from the power control mechanism; hence, the interference from cellular users to D2D users would be more critical to the D2D operation. Similarly, in the second time slot, the BS transmits messages to UEC, while UE2 sends messages to UE1 via D2D link directly. In this slot, the BS causes interference to UE1. Note that the interference at each user for single-cell and multi-cell case are various. In a multi-cell scenario, UE2 receives the interference from the cellular user not only in cell1 but also in cell 2 during the first time slot. In the second time slot, the interference caused to $\mathrm{UE} 1$ comes from both BS1 and BS2. In this case, note that $d_{12}=d_{21}=2 \beta d_{\mathrm{BD}}, d_{12}^{-\alpha}=d_{21}^{-\alpha}=(2 \beta)^{-\alpha} g$. We then give the ergodic achievable sum-rate of MODE2 in single-cell and multi-cell scenarios, respectively.

The ergodic achievable sum-rate of direct D2D communication mode for the single-cell scenario can be easily obtained as

$$
\begin{aligned}
R_{\text {sum,dir }}^{s}(\beta) \approx & \frac{1}{2}\left[\log \left(1+\frac{P_{D} \beta^{-\alpha} g}{2^{\alpha}\left(P_{C} d_{c 2}^{-\alpha}+N_{0}\right)}\right)\right. \\
& \left.+\log \left(1+\frac{P_{D} \beta^{-\alpha} g}{2^{\alpha}\left(P_{B} d_{B 1}^{-\alpha}+N_{0}\right)}\right)\right] .
\end{aligned}
$$

Similar to the analysis above, we can approximate the ergodic achievable sum-rate of direct D2D communication mode for the multi-cell scenario as follows:

$$
\begin{aligned}
R_{\text {sum,dir }}^{m}(\beta) \approx & \frac{1}{2}\left[\log \left(1+\frac{P_{D} \beta^{-\alpha} g}{2^{\alpha}\left(P_{C} d_{C_{1} 2}^{-\alpha}+P_{C} d_{C_{2} 2}^{-\alpha}+N_{0}\right)}\right)\right. \\
& \left.+\log \left(1+\frac{P_{D} \beta^{-\alpha} g}{2^{\alpha}\left(P_{B} d_{B_{1} 1}^{-\alpha}+P_{B} d_{B_{2} 1}^{-\alpha}+N_{0}\right)}\right)\right] .
\end{aligned}
$$

\subsection{MODE3: two-way DF-relayed D2D communication mode}

In MODE3, the D2D users exchange messages with each other via a two-way DF relay node located midway of D2D users, as shown in MODE3 of Figures 1 and 2. During the first time slot, D2D users transmit their messages to the relay node simultaneously. Meanwhile, the cellular user transmits messages to the BS. Thus, the relay node (UE3) receives the message influenced by UEC. After the decoding process, the relay node re-encodes the messages and retransmits it to D2D users in the second time slot. At the same time, the BS sends the message to the cellular user and causes interference to both UE1 and UE2. Similar with MODE2, the difference between the single-cell scenario and multi-cell scenario is also the source of interference. In the multi-cell scenario, users receive interference from both cells, while the single-cell scenario's interference is caused within its own cell.

We denote the channel coefficient between UE1 and UE3 as $h_{1}$ as the reciprocity of the channel. Similarly, the channel coefficient of UE2-UE3 link is denoted as $h_{2}$. For the multi-cell scenario, according to the received signal at the relay node during the first time slot, the SINR for UE1-UE3 link and the UE2-UE3 link can be obtained as

$$
\gamma_{l 3}^{m}=\frac{P_{D} \beta^{-\alpha} g\left|h_{l}\right|^{2}}{P_{C} d_{C_{1} 3}^{-\alpha}\left|h_{C_{1} 3}\right|^{2}+P_{C} d_{C_{2} 3}^{-\alpha}\left|h_{C_{2} 3}\right|^{2}+N_{0}},(l=1,2) .
$$

The SINR at the relay node can be expressed as

$$
\gamma_{\mathrm{mac}}^{m}=\frac{P_{D} \beta^{-\alpha} g\left(\left|h_{1}\right|^{2}+\left|h_{2}\right|^{2}\right)}{P_{C} d_{C_{1} 3}^{-\alpha}\left|h_{C_{1} 3}\right|^{2}+P_{C} d_{C_{2} 3}^{-\alpha}\left|h_{C_{2} 3}\right|^{2}+N_{0}} .
$$

For the second time slot, we have

$$
\gamma_{3 k}^{m}=\frac{P_{D} \beta^{-\alpha} g\left|h_{k}\right|^{2}}{P_{B} d_{B_{1} k}^{-\alpha}\left|h_{B_{1} k}\right|^{2}+P_{B} d_{B_{2} k}^{-\alpha}\left|h_{B_{2} k}\right|^{2}+N_{0}},(k=1,2) .
$$

Based on the received SINR for each link, the ergodic achievable sum-rate of MODE3 is given by

$$
R_{\mathrm{sum}, \mathrm{DF}}^{m}(\beta)=\min \left(R_{\mathrm{mac}}^{m}(\beta), R_{1, \mathrm{DF}}^{m}(\beta)+R_{2, \mathrm{DF}}^{m}(\beta)\right),
$$


where

$$
R_{1, \mathrm{DF}}^{m}(\beta)=\frac{1}{2} \min \left\{E\left\{\log \left(1+\gamma_{13}^{m}\right)\right\}, E\left\{\log \left(1+\gamma_{32}^{m}\right)\right\}\right\},
$$

$$
R_{2, \mathrm{DF}}^{m}(\beta)=\frac{1}{2} \min \left\{E\left\{\log \left(1+\gamma_{23}^{m}\right)\right\}, E\left\{\log \left(1+\gamma_{31}^{m}\right)\right\}\right\}
$$

and

$$
R_{\mathrm{mac}}^{m}(\beta)=\frac{1}{2} E\left\{\log \left(1+\gamma_{\mathrm{mac}}^{m}\right)\right\} .
$$

The ergodic achievable sum-rate of MODE3 for the single-cell scenario can be obtained in the same way. By assuming $d_{B_{1} 2} \approx d_{B_{2} 1}$, we can easily derive the ergodic achievable sum-rate of the two-way DF-relayed D2D communication mode for both single-cell and multi-cell scenarios as follows:

Theorem 1. The ergodic achievable sum-rate of MODE3 for both single-cell and multi-cell scenarios are given by

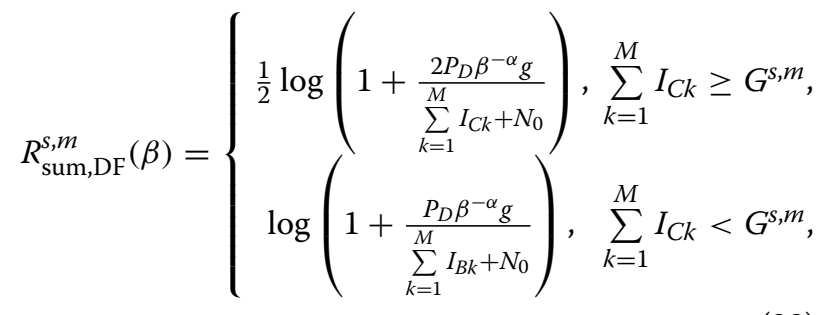

where

$$
G^{s, m}=\frac{2\left(\sum_{k=1}^{M} I_{B k}+N_{0}\right)^{2}}{2\left(\sum_{k=1}^{M} I_{B k}+N_{0}\right)+P_{D} \beta^{-\alpha} g}-N_{0},
$$

$I_{C k}$ and $I_{B k}$ represent the interference from the cellular user and the BS in cell $k$, respectively. Note that $M=1$ indicates the ergodic achievable sum-rate of the single-cell scenario with $I_{C}=P_{C} d_{c 3}^{-\alpha}$ and $I_{B}=P_{B} d_{B D}^{-\alpha}$. For the multi-cell case (i.e., $M=2$ ), we have

$$
\sum I_{C}=P_{C} d_{C_{1} 3}^{-\alpha}+P_{C} d_{C_{2} 3}^{-\alpha}
$$

and

$$
\sum I_{B}=P_{B} d_{B_{1} 2}^{-\alpha}+P_{B} d_{B_{2} 2}^{-\alpha} .
$$

Proof. See the Appendix.

Note that (28) presents an exact characterization of the ergodic achievable sum-rate performance for the D2D link. Unlike the former two transmission modes, the achievable sum-rate of MODE3 has different expressions for varies interference from cellular users, using $G^{s, m}$ as the threshold. Also, it is evident that the ergodic achievable sum-rate behavior of D2D communication depends on several major factors including the interference from cellular users $\sum_{k=1}^{M} I_{C k}$ and the BS $\sum_{k=1}^{M} I_{B k}$, the distance between the BS and D2D user $g$, the distance between D2D users $\beta$, the noise $N_{0}$ and the transmission power of the D2D user $P_{D}$. According to (28), we find that the ergodic achievable sum-rate decrease as the distance between D2D users increases, or the interference from cellular users increases, or the interference from BS increases.

\section{Transmission mode switching strategy}

In this section, we derive the transmission mode switching strategy to select the best transmission mode for the D2D communication system. Based on the ergodic achievable sum-rates derived in the previous parts, we find the crossing points of different transmission modes to maximize the system ergodic achievable sum-rate. Note that the mode switching strategies are similar for the single-cell and multi-cell scenarios. We will describe the procedure of the mode switching strategy for the multi-cell scenario in details, and then we will describe the strategy in the single-cell scenario briefly.

For the multi-cell scenario, the ergodic achievable sumrate of the traditional cellular communication mode is shown in (18). As the D2D users are close enough to each other, it is assumed that $\sum I_{C}=P_{C} d_{C_{1} 2}^{-\alpha}+P_{C} d_{C_{2} 2}^{-\alpha} \approx$ $P_{C} d_{C_{1} 3}^{-\alpha}+P_{C} d_{C_{2} 3}^{-\alpha}$. Therefore, the ergodic achievable sumrates in (20) and (28) can be written as

$$
\begin{aligned}
R_{\text {sum,dir }}^{m}= & \frac{1}{2} \log \left(1+\frac{P_{D} \beta^{-\alpha} g}{2^{\alpha}\left(\sum I_{C}+N_{0}\right)}\right) \\
& +\frac{1}{2} \log \left(1+\frac{P_{D} \beta^{-\alpha} g}{2^{\alpha}\left(\sum I_{B}+N_{0}\right)}\right)
\end{aligned}
$$

and

$$
R_{\mathrm{sum}, \mathrm{DF}}^{m}= \begin{cases}\frac{1}{2} \log \left(1+\frac{2 P_{D} \beta^{-\alpha} g}{\sum I_{C}+N_{0}}\right), \quad \sum I_{C} \geq G^{m}, \\ \log \left(1+\frac{P_{D} \beta^{-\alpha} g}{\sum I_{B}+N_{0}}\right), \quad \sum I_{C}<G^{m} .\end{cases}
$$

Note that $\sum I_{C}$ is short for $\sum_{k=1}^{2} I_{C k}$, and $\sum I_{B}$ is short for $\sum_{k=1}^{2} I_{B k}$. From (18), (32), and (33), it is obvious that the expressions of ergodic achievable sum-rate for MODE1 and MODE2 are fixed, while the expression for MODE3 is related with the interference level from cellular users $\sum I_{C}$. Therefore, we discuss the transmission mode switching strategy in two cases: $\sum I_{C} \geq G^{m}$ and $\sum I_{C}<G^{m}$. 
4.1 The case of $\sum I_{C} \geq G^{m}$

With $\sum I_{C} \geq G^{m}$, the expression of the ergodic achievable sum-rate for MODE3 is given by

$$
R_{\text {sum }, \mathrm{DF}}^{m}=\frac{1}{2} \log \left(1+\frac{2 P_{D} \beta^{-\alpha} g}{\sum I_{C}+N_{0}}\right) .
$$

First, we compare the ergodic achievable sum-rate of MODE1 and MODE3. To compare the ergodic achievable sum-rate of two transmission modes, MODE1 and MODE3, we subtract the ergodic achievable sum-rate of MODE1 from the ergodic achievable sum-rate of MODE3 (i.e., $R_{\text {sum }, \mathrm{DF}}^{m}-R_{\text {sum,tra }}^{m}$ ), and let the result be above zero to get the threshold of $\beta$. Therefore, when $\beta$ is above the threshold, we choose one transmission mode; otherwise, switch to the other transmission mode. We subtract (18) from (34) to obtain

$$
\begin{aligned}
R_{\text {sum }, \mathrm{DF}}^{m}-R_{\text {sum }, \text { tra }}^{m}= & \frac{1}{2} \log \left(1+\frac{2 P_{D} \beta^{-\alpha} g}{\sum I_{C}+N_{0}}\right) \\
& -\left[\frac{1}{2} \log \left(1+\frac{P_{D} g}{P_{D} d_{2 B_{1}}^{-\alpha}+N_{0}}\right)\right. \\
& \left.+\frac{1}{2} \log \left(1+\frac{P_{D} g}{P_{D} d_{1 B_{2}}^{-\alpha}+N_{0}}\right)\right],
\end{aligned}
$$

and let

$$
\begin{aligned}
P=1+\frac{2 P_{D} \beta^{-\alpha} g}{\sum I_{C}+N_{0}}- & \left(1+\frac{P_{D} g}{P_{D} d_{2 B_{1}}^{-\alpha}+N_{0}}\right) \\
& \times\left(1+\frac{P_{D} g}{P_{D} d_{1 B_{2}}^{-\alpha}+N_{0}}\right) .
\end{aligned}
$$

For $P>0$, it can be derived that

$$
\beta<Q_{1}^{m},
$$

where

$$
Q_{1}^{m}=\left(\frac{2\left(P_{D} d_{2 B_{1}}^{-\alpha}+N_{0}\right)\left(P_{D} d_{1 B_{2}}^{-\alpha}+N_{0}\right)}{\left(\sum I_{C}+N_{0}\right)\left(P_{D} d_{2 B_{1}}^{-\alpha}+P_{D} d_{1 B_{2}}^{-\alpha}+P_{D} g+2 N_{0}\right)}\right)^{\frac{1}{\alpha}} .
$$

Based on the results in (37) and (38), MODE3 is chosen when $\beta<Q_{1}^{m}$. Similarly, MODE1 is selected for $\beta \geq Q_{1}^{m}$. When $\beta \geq Q_{1}^{m}$, we can get that $P \leq 0$ and $R_{\text {sum, DF }}^{m} \leq R_{\text {sum,tra }}^{m}$. Therefore, the transmission mode switching strategy between MODE1 and MODE3 can be given by

$$
\left\{\begin{array}{l}
\beta<Q_{1}^{m}, \text { MODE3; } \\
\beta \geq Q_{1}^{m}, \text { MODE1. }
\end{array}\right.
$$

As the parameter $\beta$ indicates the distance between the D2D users, it reveals that MODE1 outperforms MODE3 in ergodic achievable sum-rate performance when the distance between the D2D users is long enough. It is reasonable that for a nearer D2D communication pairs, the
BS control the transmit power of D2D users to restrain the interference to the cellular user. However, for the users far from each other, traditional cellular communication mode takes an advantage as the transmit power of the users is limited.

Using the same methods, we subtract (32) from (34) to get the switching point for MODE2 and MODE3. After some calculations, we have

$$
\left\{\begin{array}{l}
\beta \geq Q_{2}^{m}, \text { MODE3; } \\
\beta<Q_{2}^{m}, \text { MODE2, }
\end{array}\right.
$$

where

$Q_{2}^{m}=\left(\frac{P_{D} g}{2^{2 \alpha+1}\left(\sum I_{B}+N_{0}\right)-2^{\alpha}\left(\sum I_{C}+\sum I_{B}+2 N_{0}\right)}\right)^{\frac{1}{\alpha}}$.

For the D2D users located not far from each other, (40) and (41) give the mode selection strategy. It is well known that the relay-assisted communication has great potential in enhancing the system performance, especially when there is no direct connection between the source and the destination (for example, due to shadowing or long distance). Therefore, when $\beta$ is higher than a particular threshold, the two-way DF-relayed D2D communication mode achieves higher ergodic achievable sum-rate than that of the direct D2D communication mode.

According to the results in (39) and (40), we have the overall transmission mode switching strategy under the circumstance of $\sum I_{C} \geq G^{m}$ as follows:

$$
\left\{\begin{array}{cc}
\beta<Q_{2}^{m}, & \text { MODE2; } \\
Q_{2}^{m} \leq \beta<Q_{1}^{m}, & \text { MODE3; } \\
\beta \geq Q_{1}^{m}, & \text { MODE1. }
\end{array}\right.
$$

From the above results, we can see that under the circumstances of relatively stronger interference, MODE1 is more suitable to be chosen when there is a long distance between the D2D users. MODE2 is preferred when the D2D users are close enough to each other. Note that this strategy is a result of strong interference from the cellular users, but the outcome is a little bit different if the interference is weak, which can be seen from the analysis in the next section.

\subsection{The case of $\sum I_{C}<G^{m}$}

Similar to the derivation in Section 4.1, we first compare the ergodic achievable sum-rate of MODE1 and MODE3. In the case of $\sum I_{C}<G^{m}$, the ergodic achievable sumrate for MODE3 can be written as

$$
R_{\mathrm{sum}, \mathrm{DF}}^{m}=\log \left(1+\frac{P_{D} \beta^{-\alpha} g}{\sum I_{B}+N_{0}}\right) .
$$


Subtract it from (18), and after some calculation, we can get

$$
\left\{\begin{array}{l}
\beta<Q_{3}^{m}, \text { MODE3; } \\
\beta \geq Q_{3}^{m}, \text { MODE1, }
\end{array}\right.
$$

where

$$
\begin{aligned}
Q_{3}^{m} & =\left[\frac{-B_{1}+\sqrt{D_{1}}}{2 A_{1}}\right]^{-\frac{1}{\alpha}} \\
A_{1} & =\left(\frac{P_{D} g}{\sum I_{B}+N_{0}}\right)^{2} \\
B_{1} & =\frac{2 P_{D} g}{\sum I_{B}+N_{0}}, \\
C_{1} & =1-\left(1+\frac{P_{D} g}{P_{D} d_{2 B_{1}}^{-\alpha}+N_{0}}\right)\left(1+\frac{P_{D} g}{P_{D} d_{1 B_{2}}^{-\alpha}+N_{0}}\right)
\end{aligned}
$$

and

$$
D_{1}=B_{1}^{2}-4 A_{1} C_{1} .
$$

The strategy of switching between MODE1 and MODE3 in (44) is the same as (39), which means the traditional cellular transmission communication mode always outperforms the two-way DF-relayed D2D communication mode for long distance between the D2D users, no matter what the interference level is.

Then we compare MODE2 and MODE3 using the same method, by comparing (32) and (43), we have

$$
\begin{aligned}
T= & \left(1+\frac{P_{D} \beta^{-\alpha} g}{\sum I_{B}+N_{0}}\right)^{2}-\left(1+\frac{P_{D} \beta^{-\alpha} g}{2^{\alpha}\left(\sum I_{C}+N_{0}\right)}\right) \\
& \times\left(1+\frac{P_{D} \beta^{-\alpha} g}{2^{\alpha}\left(\sum I_{B}+N_{0}\right)}\right) .
\end{aligned}
$$

Let $T \geq 0$. After some manipulations, we have

$$
\left\{\begin{array}{l}
\text { when } \sum I_{C}>H^{m}, \beta \geq Q_{4}^{m} ; \\
\text { when } \sum I_{C}=H^{m}, \quad \beta=\emptyset ; \\
\text { when } \sum I_{C}<H^{m}, \beta \leq Q_{4}^{m},
\end{array}\right.
$$

where

$$
H^{m}=\frac{\sum I_{B}+N_{0}}{2^{\alpha+1}-1}-N_{0}
$$

and

$Q_{4}^{m}=\left(\frac{\left[2^{2 \alpha}\left(\sum I_{C}+N_{0}\right)-\left(\sum I_{B}+N_{0}\right)\right] P_{D} g}{2^{\alpha}\left(\sum I_{B}+N_{0}\right)\left[\sum I_{C}+2 N_{0}+\sum I_{B}-2^{\alpha+1}\left(\sum I_{C}+N_{0}\right)\right]}\right)^{\frac{1}{\alpha}}$.

When $\sum I_{C}=H^{m}, \beta=\emptyset$ means that in this situation, $T<0$, and $R_{\text {sum,dir }}^{m}$ is always bigger than $R_{\text {sum,DF }}^{\mathrm{m}}$; thus, we choose MODE2. But in the other two cases, $R_{\text {sum,DF }}^{\mathrm{m}} \geq R_{\text {sum,dir, }}^{m}$, thus we choose MODE3. We get the result as below:

$$
\left\{\begin{array}{l}
\sum I_{C}<H^{m},\left\{\begin{array}{l}
\beta>Q_{4}^{m}, \text { MODE2 } \\
\beta \leq Q_{4}^{m}, \text { MODE3 }
\end{array}\right. \\
\sum I_{C}=H^{m}, \begin{array}{c}
M O D E 2 \\
\sum I_{C}>H^{m}
\end{array},\left\{\begin{array}{l}
\beta \geq Q_{4}^{m}, \text { MODE3 } \\
\beta<Q_{4}^{m}, \text { MODE2 }
\end{array}\right.
\end{array}\right.
$$

Notice that when $\sum I_{C}=H^{m}$, the value of $Q_{4}^{m}$ tend to be infinite, which means this case can be combined into the case of $\sum I_{C}>H^{m}$. As a result, the strategy above can be simplified as follows:

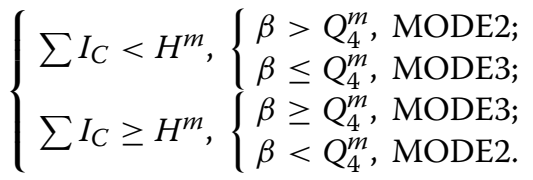

From the above results, we can see that, we need to further discuss the case of different interference level to determine the switch point between MODE2 and MODE3. While the interference from the cellular users is extremely low, i.e., $\sum I_{C}<H^{m}(H$ is obviously smaller than $G$ ), we choose MODE3 as the distance between the D2D users is short, and switch to MODE2 if $\beta$ is above the threshold $Q_{4}^{m}$. From the viewpoint of reality, this phenomenon comes from the fact that the long distance make hard for the D2D communication mode. In this scenario, either the direct D2D communication mode or two-way DF-relayed D2D communication model shows its advantages.

To achieve the complete switching strategy, we finally come to the comparison of MODE1 and MODE2. Subtracting (18) from (32), and after some calculation it yields

$$
\left\{\begin{array}{l}
\beta<Q_{5}^{m}, M O D E 2 \\
\beta \geq Q_{5}^{m}, M O D E 1
\end{array}\right.
$$

where

$$
\begin{aligned}
Q_{5}^{m} & =\left[\frac{-B_{2}+\sqrt{D}_{2}}{2 A_{2}}\right]^{-\frac{1}{\alpha}} \\
A_{2} & =\frac{\left(P_{D} g\right)^{2}}{2^{2 \alpha}\left(\sum I_{B}+N_{0}\right)\left(\sum I_{C}+N_{0}\right)} \\
B_{2} & =\frac{P_{D} g\left(\sum I_{B}+\sum I_{C}+2 N_{0}\right)}{2^{\alpha}\left(\sum I_{B}+N_{0}\right)\left(\sum I_{C}+N_{0}\right)} \\
C_{2} & =1-\left(1+\frac{P_{D} g}{P_{D} d_{2 B_{1}}^{-\alpha}+N_{0}}\right)\left(1+\frac{P_{D} g}{P_{D} d_{1 B_{2}}^{-\alpha}+N_{0}}\right)
\end{aligned}
$$

and

$$
D_{2}=B_{4}^{2}-4 A_{4} C_{4} .
$$


From (44), (55), and (56), we can obtain the following transmission mode switching strategy under the circumstance of $\sum I_{C}<G^{m}$ :

$$
\sum I_{C}<H^{m},\left\{\begin{array}{cc}
\beta \leq Q_{4}^{m}, & \text { MODE3; } \\
Q_{4}^{m}<\beta<Q_{5}^{m}, & \text { MODE2; } \\
\beta \geq Q_{5}^{m}, & \text { MODE1; } \\
\beta<Q_{4}^{m}, & \text { MODE2; } \\
Q_{4}^{m} \leq \beta<Q_{3}^{m}, & \text { MODE3; } \\
\beta \geq Q_{3}^{m}, & \text { MODE1 }
\end{array}\right.
$$

Compared with the switching strategy in Section 4.1, it is obvious that even if the interference is weak, MODE1 also has the best ergodic achievable sum-rate performance for the far-apart D2D users. When the D2D users are close enough to each other, MODE3 outperforms MODE2 if the interference from cellular users is weaker than $H^{m}$, but it will lose its advantage as the interference gets a little stronger. Notice that the switching result of $H^{m}<\sum I_{C}<$ $G^{m}$ is the same as $\sum I_{C} \geq G^{m}$ with different thresholds (i.e., the value of $Q^{m}$ ).

\subsection{The overall switching strategy in both single-cell and multi-cell scenarios}

Taking both results in Section 4.1 and Section 4.2, the final transmission mode switching strategy for the multicell scenario is obtained. The result is not only suitable for the multi-cell scenario, but also suitable for the single-cell scenario as the procedure of derivation is similar. Therefore, the overall switching strategy for both single-cell and multi-cell scenarios is shown as follows with different thresholds (i.e., $Q_{1}$ to $Q_{5}$ ):

$$
\begin{array}{r}
\sum_{k=1}^{M} I_{C k}<H^{i},\left\{\begin{array}{cc}
\beta \leq Q_{4}^{i}, & \text { MODE3; } \\
Q_{4}^{i}<\beta<Q_{5}^{i}, & \text { MODE2; } \\
\beta \geq Q_{5}^{i}, & \text { MODE1; }
\end{array}\right. \\
H^{i} \leq \sum_{k=1}^{M} I_{C k}<G^{i},\left\{\begin{array}{cc}
\beta<Q_{4}^{i}, & \text { MODE2; } \\
Q_{4}^{i} \leq \beta<Q_{3}^{i}, & \text { MODE3; } \\
\beta \geq Q_{3}^{i}, & \text { MODE1; }
\end{array}\right. \\
\sum_{k=1}^{M} I_{C k} \geq G^{i},\left\{\begin{array}{cc}
\beta<Q_{2}^{i}, & \text { MODE2; } \\
Q_{2}^{i} \leq \beta<Q_{1}^{i}, & \text { MODE3; } \\
\beta \geq Q_{1}^{i}, & \text { MODE1, }
\end{array}\right.
\end{array}
$$

where $M=1,2$ and $i=s, m$. For the single-cell scenario $M=1, i=s$, while $M=2, i=m$ refer to the multi-cell scenario. $Q_{1}^{s} \sim Q_{5}^{s}$ and $H^{s}$ is shown as follows:

$$
\begin{aligned}
& Q_{1}^{s}=\left(\frac{2 N_{0}}{I_{C}+N_{0}}\right)^{\frac{1}{\alpha}}, \\
& Q_{2}^{s}=\left(\frac{P_{D} g}{2^{2 \alpha+1}\left(I_{B}+N_{0}\right)-2^{\alpha}\left(I_{C}+I_{B}+2 N_{0}\right)}\right)^{\frac{1}{\alpha}},
\end{aligned}
$$

$$
\begin{aligned}
Q_{3}^{s} & =\left[\frac{-B_{3}+\sqrt{D_{3}}}{2 A_{3}}\right]^{-\frac{1}{\alpha}} \\
A_{3} & =\left(\frac{P_{D} g}{I_{B}+N_{0}}\right)^{2} \\
B_{3} & =\frac{2 P_{D} g}{I_{B}+N_{0}} \\
C_{3} & =-\frac{P_{D} g}{N_{0}} \\
D_{3} & =B_{3}^{2}-4 A_{3} C_{3}, \\
Q_{4}^{s}= & \left(\frac{\left[2^{2 \alpha}\left(I_{C}+N_{0}\right)-\left(I_{B}+N_{0}\right)\right] P_{D} g}{2^{\alpha}\left(I_{B}+N_{0}\right)\left[I_{C}+2 N_{0}+I_{B}-2^{\alpha+1}\left(I_{C}+N_{0}\right)\right]}\right) \\
Q_{5}^{s} & =\left[\frac{-B_{4}+\sqrt{D_{4}}}{2 A_{4}}\right]^{-\frac{1}{\alpha}} \\
A_{4} & =\frac{\left(P_{D} g\right)^{2}}{2^{2 \alpha}\left(I_{B}+N_{0}\right)\left(I_{C}+N_{0}\right)}, \\
B_{4} & =\frac{P_{D} g\left(I_{B}+I_{C}+2 N_{0}\right)}{2^{\alpha}\left(I_{B}+N_{0}\right)\left(I_{C}+N_{0}\right)} \\
C_{4} & =-\frac{P_{D} g}{N_{0}}, \\
D_{4} & =B_{4}^{2}-4 A_{4} C_{4}, \\
H^{s} & =\frac{I_{B}+N_{0}}{2^{\alpha+1}-1}-N_{0} .
\end{aligned}
$$

The final transmission mode switching strategy reveals that MODE1 always has the best performance with a long distance between D2D users for all the interference levels. We can observe that when the interference is stronger than $H^{i}$, the two transmission mode switching strategies remain the same, in the order of MODE2, MODE3, and MODE1 as $\beta$ increases. But note that these two cases use different thresholds (i.e., the value of $Q$ ) to switch among three transmission modes. When the interference is weak, the transmission switching strategy turns to a different strategy in the order of MODE3, MODE2, and MODE1. To sum up, we present the mode switching scheme shown in Figure 3.

\section{Numerical results}

In this section, we present numerical results to illustrate the improvement of the system performance with the strategy of transmission mode switching. For convenience, the distance between D2D user and the BS is normalized to ten times the unity. Since D2D communications generally consider a pair of nearby users, we have $d_{B 1} \approx d_{B 2}$ for the single-cell scenario and $d_{B_{1} 1} \approx d_{B_{2} 2}$ for the multi-cell scenario. We set the transmit power of the 


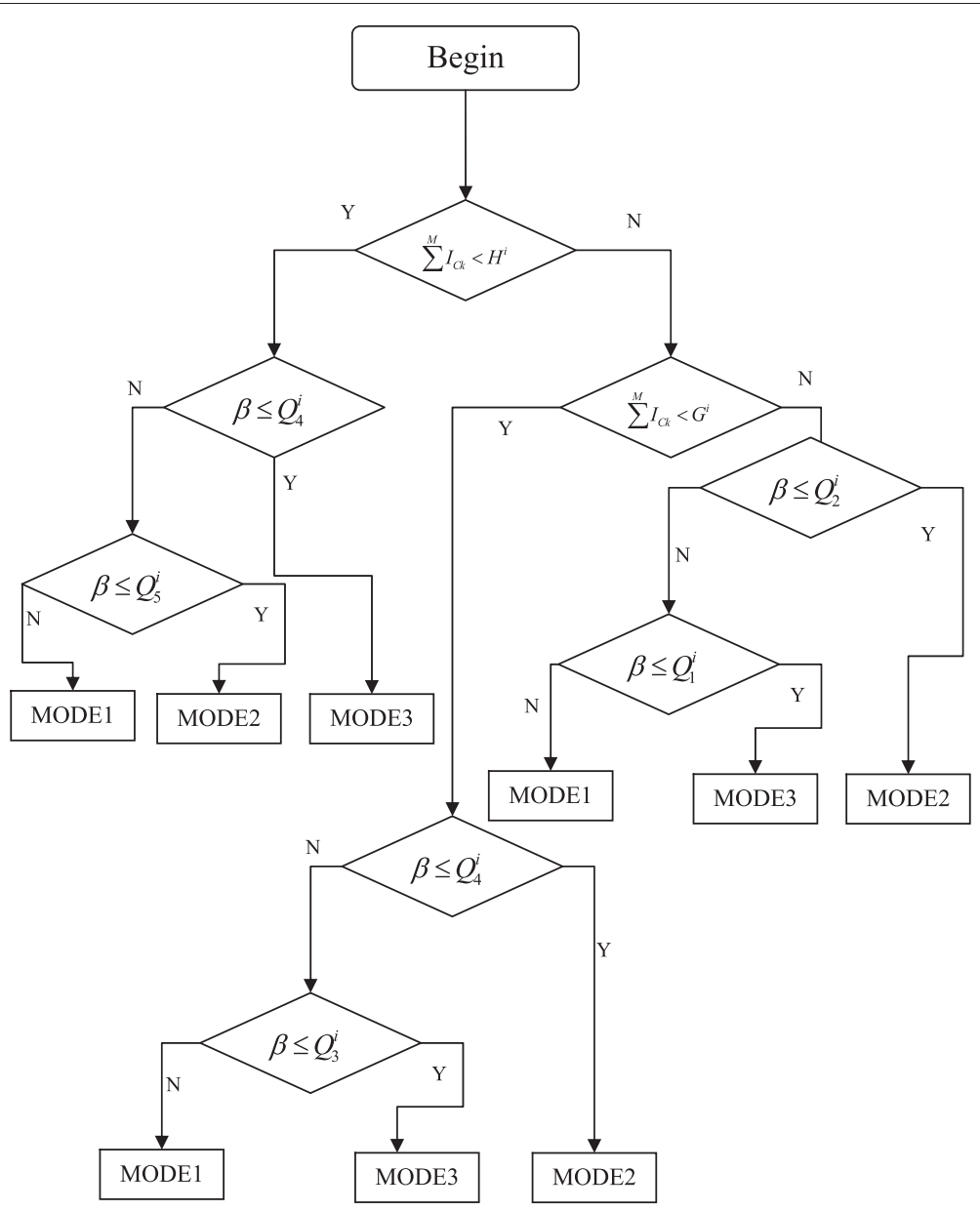

Figure 3 The flow chart of the transmission switching scheme between three modes for the D2D communication system.

BS as $P_{B}=100$ while the transmit power of each user is assumed to be equal that $P_{C}=P_{D}=1$. The interference from the cellular user varies with the location of cellular user. Note that all the Rayleigh fading channels are independent and identically distributed. The channel gains are modeled as $h_{i j}$ with $h_{i j} \sim C N(0,1)$.

The figures in this section show the ergodic achievable sum-rates of three transmission modes and the corresponding switching result under different interference level versus $d_{12}$ (distance between D2D users) or $d_{\mathrm{BD}}$ (distance between the BS and D2D user). The left part of each figure illustrates the performance for the single-cell scenario, while the right part shows the multi-cell case.

Figure 4 shows the switching scheme results for the strong interference from cellular user (i.e., $I_{C}>G$ ). For both single-cell and multi-cell scenarios, the ergodic achievable sum-rate of MODE2 and MODE3 decrease as the distance between D2D users increases while the ergodic achievable sum-rate of MODE1 maintains the same level as the communication completed via base station. It is obvious that when the D2D users are close enough, the ergodic achievable sum-rate of MODE2 is higher than the other two modes. It reveals that the direct D2D communication strategy outperforms the two-way relayed D2D communication strategy for the close D2D communication pairs or a strong interference level. However, the result is the opposite when the interference is weak, as Figure 5 shows. The traditional cellular communication strategy takes its advantage over the other two modes when the distance between D2D users is far enough. The curves in Figure 4 verify the analytical results in Section 4.

Figure 6 plots the mode switching strategy for the middle interference level. The tendency of the ergodic achievable sum-rate for the three transmission modes is the same as in Figure 4. From Figure 6, we can see that the proposed transmission mode switching strategy always selects a transmission mode which has the highest ergodic achievable sum-rate value among all transmission modes.

Figure 5 illustrates the case of weak interference from cellular users. It can be easily found that the switching strategy is different from the above two cases. We 

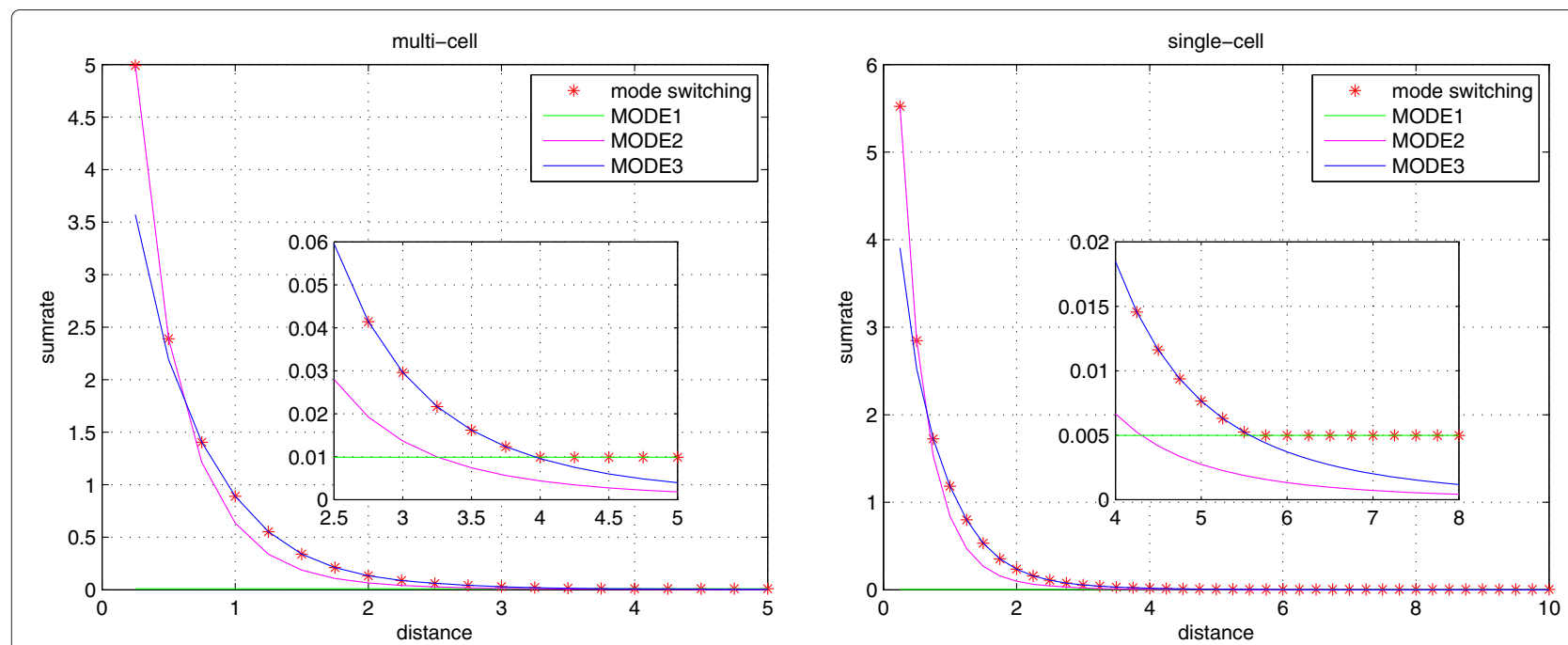

Figure 4 The ergodic achievable sum-rate of three transmission modes and corresponding switching results for the case of $\sum I_{C}>G$ versus $d_{12}$.

also notice that in the single-cell scenario of Figure 5, the transmission mode switching result only involves two transmission modes, that is because $Q_{4}<0$ in this case. The ergodic achievable sum-rates of switching strategy plotted in these figures show the accuracy of our analytical results. The numerical results demonstrate that by applying the transmission mode switching strategy, the D2D communication underlaying cellular system achieves a remarkable enhancement of ergodic achievable sumrate compared to the conventional ways of using only one transmission mode, for both single-cell and multi-cell scenarios.
From Figures 4, 5, 6, we notice that, by fixing the distance between $\mathrm{BS}$ and $\mathrm{D} 2 \mathrm{D}$ users $\left(d_{\mathrm{BD}}\right)$, the ergodic achievable sum-rate of MODE1 maintains the same as the distance changes. Figure 7 represents the achievable sumrate for the fixed $d_{12}$ and various $d_{\mathrm{BD}}$. From Figure 7 it is shown that, the ergodic achievable sum-rate of MODE2 and MODE3 increases slightly with $d_{\mathrm{BD}}$, while the ergodic achievable sum-rate of MODE1 decreases. When the D2D users are close to the base station, the traditional cellular transmission mode achieves better performance than the other two transmission modes. For the D2D users located at the edge of the cell, MODE3 takes the optimal
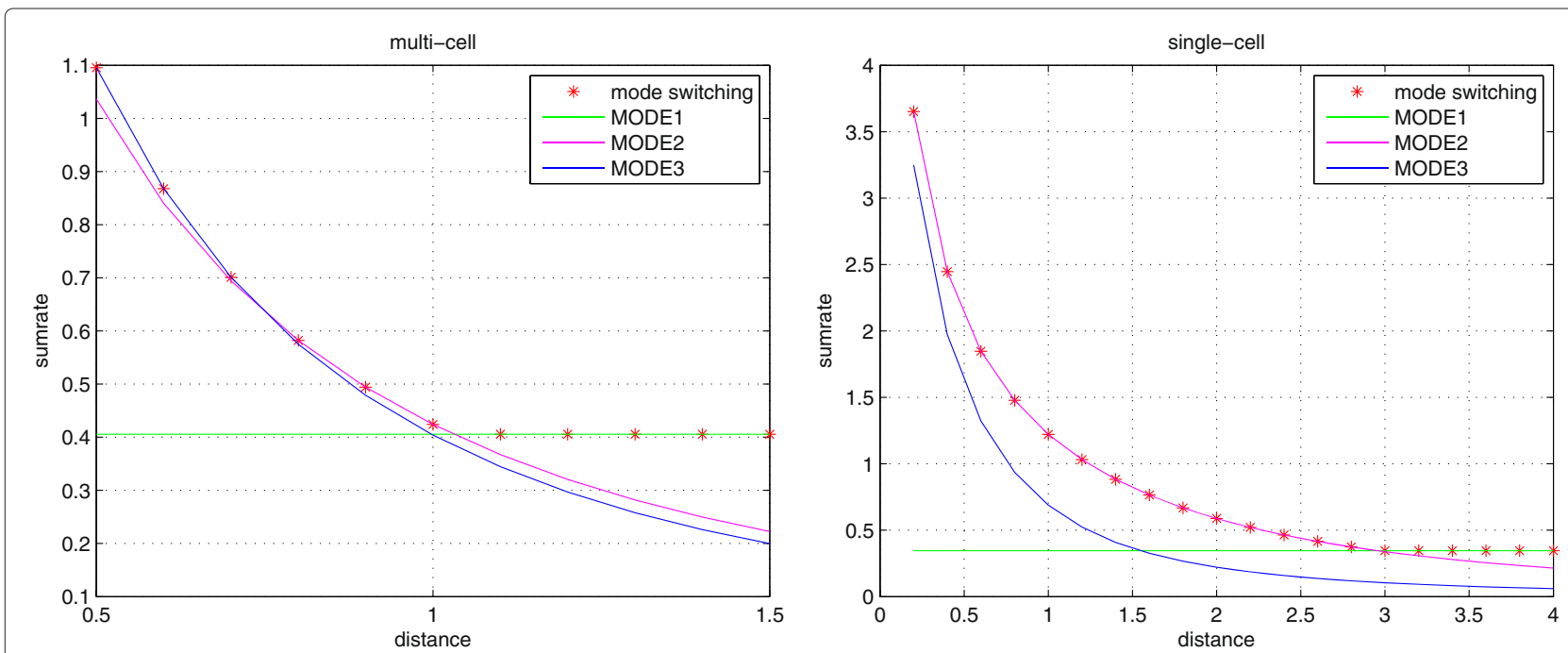

Figure 5 The ergodic achievable sum-rate of three transmission modes and corresponding switching results for the case of $\sum I_{C}<H$ versus $d_{12}$. 

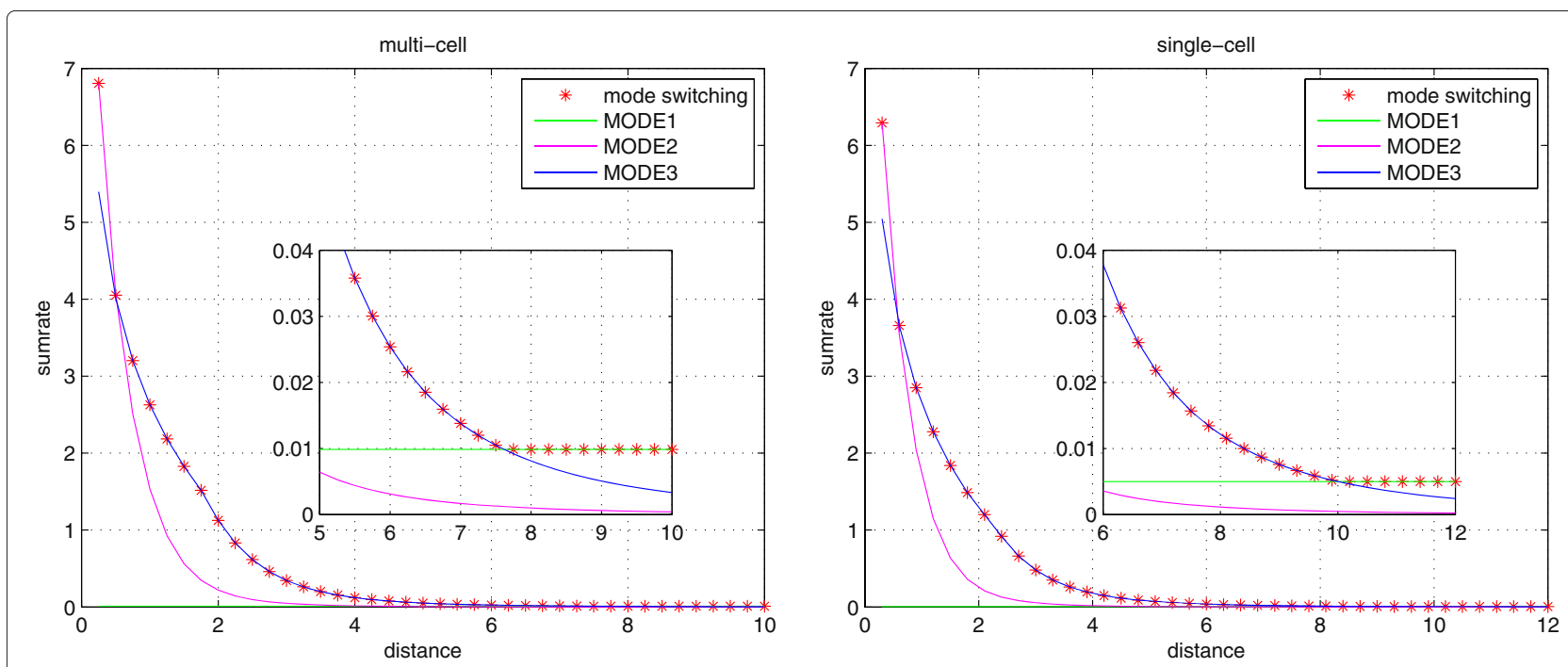

Figure 6 The ergodic achievable sum-rate of three transmission modes and switching results for the case of $H<\sum I_{C}<G$ versus $d_{12}$.

performance. Nevertheless, the proposed transmission mode switching strategy always has the best performance for the different location of the D2D users in the cell.

\section{Conclusion}

This paper presented a transmission mode switching strategy for D2D communication underlaying cellular networks in both single-cell and multi-cell scenarios. We first introduced three communication transmission modes in these two scenarios, which are the traditional cellular communication mode, direct D2D communication mode, and two-way DF-relayed D2D communication mode. By deriving the expression for the ergodic achievable sumrate of these transmission modes, we got the threshold of different transmission modes to attain maximum sumrate of the system. Based on this, we got the transmission mode switching strategy theoretically. Simulation results showed that by applying the transmission mode switching strategy, the ergodic achievable sum-rate of the system achieves a remarkable enhancement under different

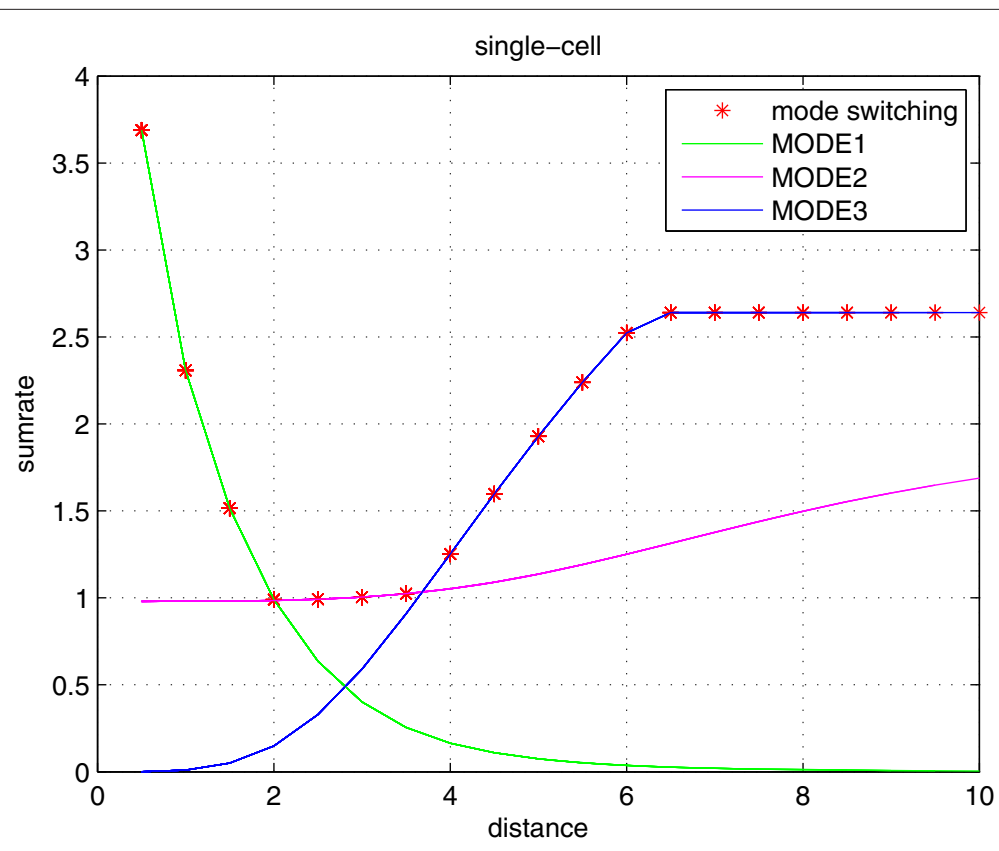

Figure 7 Transmission mode switching results versus the distance between BS and the D2D users $d_{\mathrm{BD}}$. 
interference level and distance of D2D users. The proposed transmission mode switching strategy could be an effective method for efficient D2D communication.

\section{Appendix}

\section{Proof of Theorem 1}

For the multi-cell scenario, we denote

$$
\sum I_{C}=P_{C} d_{C_{1} 3}^{-\alpha}+P_{C} d_{C_{2} 3}^{-\alpha}
$$

and

$$
\sum I_{B}=P_{B} d_{B_{1} 2}^{-\alpha}+P_{B} d_{B_{2} 2}^{-\alpha}=P_{B} d_{B_{1} 1}^{-\alpha}+P_{B} d_{B_{2} 1}^{-\alpha}
$$

Based on that, we use Jensen's inequality to write (25), (26), and (27) as

$$
\begin{aligned}
R_{1, \mathrm{DF}}^{m}(\beta) \approx & \frac{1}{2} \min \left\{\log \left(1+\frac{P_{D} \beta^{-\alpha} g}{\sum I_{C}+N_{0}}\right),\right. \\
& \left.\times \log \left(1+\frac{P_{D} \beta^{-\alpha} g}{\sum I_{B}+N_{0}}\right)\right\}, \\
R_{2, \mathrm{DF}}^{m}(\beta) \approx & \frac{1}{2} \min \left\{\log \left(1+\frac{P_{D} \beta^{-\alpha} g}{\sum I_{C}+N_{0}}\right),\right. \\
& \left.\times \log \left(1+\frac{P_{D} \beta^{-\alpha} g}{\sum I_{B}+N_{0}}\right)\right\}
\end{aligned}
$$

and

$$
R_{\mathrm{mac}}^{m}(\beta) \approx \frac{1}{2} \log \left(1+\frac{\left.2 P_{D} \beta^{-\alpha} g\right)}{\sum I_{C}+N_{0}}\right) .
$$

To simplify (24), we should determine the value of $R_{1, D F}^{m}(\beta)$ and $R_{2, D F}^{m}(\beta)$. According to the above expressions, we can get that

$$
\left\{\begin{array}{l}
\sum I_{C}>\sum I_{B}, R_{1, \mathrm{DF}}^{m}(\beta)=\frac{1}{2} \log \left(1+\frac{P_{D} \beta^{-\alpha} g}{\sum I_{C}+N_{0}}\right)=R_{2, \mathrm{DF}}^{m}(\beta) ; \\
\sum I_{C}<\sum I_{B}, R_{1, \mathrm{DF}}^{m}(\beta)=\frac{1}{2} \log \left(1+\frac{P_{D} \beta^{-\alpha} g}{\sum I_{B}+N_{0}}\right)=R_{2, \mathrm{DF}}^{m}(\beta) .
\end{array}\right.
$$

Therefore, the ergodic achievable sum-rate of MODE3 can be obtained as
First, we consider the case $\sum I_{C}>\sum I_{B}$. We denote

$$
F=\left(1+\frac{P_{D} \beta^{-\alpha} g}{\sum I_{C}+N_{0}}\right)^{2}-1-\frac{2 P_{D} \beta^{-\alpha} g}{\sum I_{C}+N_{0}},
$$

and it can be obtained that

$$
F=\left(\frac{P_{D} \beta^{-\alpha} g}{\sum I_{C}+N_{0}}\right)^{2}
$$

which is always above zero. Thus we have

$$
R_{\mathrm{sum}, \mathrm{DF}}^{m}(\beta)=\frac{1}{2} \log \left(1+\frac{2 P_{D} \beta^{-\alpha} g}{\sum I_{C}+N_{0}}\right) .
$$

Then, we consider the case $\sum I_{C}<\sum I_{B}$. In this case, we set

$$
L=1+\frac{2 P_{D} \beta^{-\alpha} g}{\sum I_{B}+N_{0}}+\left(\frac{2 P_{D} \beta^{-\alpha} g}{\sum I_{B}+N_{0}}\right)^{2}-1-\frac{2 P_{D} \beta^{-\alpha} g}{\sum I_{C}+N_{0}},
$$

and let $L>0$ to derive that

$$
\sum I_{C}>\frac{2\left(\sum I_{B}+N_{0}\right)^{2}}{2\left(\sum I_{B}+N_{0}\right)+P_{D} \beta^{-\alpha} g}-N_{0} .
$$

Under this circumstance, we know that

$$
R_{\mathrm{sum}, \mathrm{DF}}^{m}=\frac{1}{2} \log \left(1+\frac{2 P_{D} \beta^{-\alpha} g}{\sum I_{C}+N_{0}}\right) .
$$

When $\sum I_{C}<\frac{2\left(\sum I_{B}+N_{0}\right)^{2}}{2\left(\sum I_{B}+N_{0}\right)+P_{D} \beta^{-\alpha} g}-N_{0}$, we can get that

$$
R_{\mathrm{sum}, \mathrm{DF}}^{m}=\log \left(1+\frac{P_{D} \beta^{-\alpha} g}{\sum I_{B}+N_{0}}\right) .
$$

Denote $G^{m}=\frac{2\left(\sum I_{B}+N_{0}\right)^{2}}{2\left(\sum I_{B}+N_{0}\right)+P_{D} \beta^{-\alpha} g}-N_{0}$, we have the ergodic achievable sum-rate as follows:

$$
\left\{\begin{array}{l}
\sum I_{C}>G^{m}, R_{\text {sum }, \mathrm{DF}}^{m}=\frac{1}{2} \log \left(1+\frac{2 P_{D} \beta^{-\alpha} g}{\sum I_{C}+N_{0}}\right) ; \\
\sum I_{C}<G^{m}, R_{\text {sum }, \mathrm{DF}}^{m}=\log \left(1+\frac{P_{D} \beta^{-\alpha} g}{\sum I_{B}+N_{0}}\right) .
\end{array}\right.
$$

As $P_{D} \beta^{-\alpha} g>0$, we know that

$$
\frac{2\left(\sum I_{B}+N_{0}\right)^{2}}{2\left(\sum I_{B}+N_{0}\right)+0}-N_{0}>\frac{2\left(\sum I_{B}+N_{0}\right)^{2}}{2\left(\sum I_{B}+N_{0}\right)+P_{D} \beta^{-\alpha} g}-N_{0},
$$

$$
\left\{\begin{array}{l}
\sum I_{C}>\sum I_{B}, R_{\text {sum }, \mathrm{DF}}^{m}(\beta)=\min \left(\frac{1}{2} \log \left(1+\frac{\left.2 P_{D} \beta^{-\alpha} g\right)}{\sum I_{C}+N_{0}}\right), \log \left(1+\frac{P_{D} \beta^{-\alpha} g}{\sum I_{C}+N_{0}}\right)\right), \\
\sum I_{C}<\sum I_{B}, R_{\text {sum }, \mathrm{DF}}^{m}(\beta)=\min \left(\frac{1}{2} \log \left(1+\frac{\left.2 P_{D} \beta^{-\alpha} g\right)}{\sum I_{C}+N_{0}}\right), \log \left(1+\frac{P_{D} \beta^{-\alpha} g}{\sum I_{B}+N_{0}}\right)\right)
\end{array}\right.
$$


that is $\sum I_{B}>G^{m}$. From (87) and (92), we can get the ergodic achievable sum-rate of MODE3 for multi-cell scenario as follows:

$$
R_{\mathrm{sum}, \mathrm{DF}}^{m}=\left\{\begin{array}{l}
\frac{1}{2} \log \left(1+\frac{2 P_{D} \beta^{-\alpha} g}{\sum I_{C}+N_{0}}\right), \quad \sum I_{C}>G^{m} \\
\log \left(1+\frac{P_{D} \beta^{-\alpha} g}{\sum I_{B}+N_{0}}\right), \quad \sum I_{C}<G^{m} .
\end{array}\right.
$$

For the single-cell scenario, the process of derivation is similar, and replace $\sum I_{C}$ with $I_{C}, \sum I_{B}$ with $I_{B}$ in the equation above, the ergodic achievable sum-rate of MODE3 for the single-cell scenario is obtained. Combine the expressions for both scenarios, we can get Theorem 1 .

\section{Competing interests}

The authors declare that they have no competing interests.

\section{Acknowledgements}

This work of Y. Y. Ni, H. B. Zhu was supported by the National Science and Technology Major Project of Ministry of Science and Technology of China under Grant 2011ZX03005-004-03, China 973 project (26) under Grant 2013CB329005, the NSF funding of China 61171093 and Jiangsu Province Important Special Project BK2011027. The work of S. Jin and D. Qiao was supported by the National Natural Science Foundation of China under Grant 61222102 and the Natural Science Foundation of Jiangsu Province under Grant BK2012021. This work of X. Li was supported by National Natural Science Foundation of China under Grant 61101089, Specialized Research Fund for the Doctoral Program of Higher Education under Grant 20110092120014.

\section{Author details}

1 Jiangsu Key Laboratory of Wireless Communications College of Telecommunications and Information Engineering, Nanjing University of Posts and Telecommunications, Nanjing 210003, China. ${ }^{2}$ National Mobile Communications Research Laboratory, Southeast University, Nanjing 210096, China.

Received: 28 May 2014 Accepted: 25 August 2014 Published: 3 September 2014

\section{References}

1. G Fodor, E Dahlman, G Mildh, S Parkvall, N Reider, G Miklós, Z Turányi, Design aspects of network assisted device-to-device communications. IEEE Commun. Mag. 50(3), 170-177 (2012)

2. K Doppler, MP Rinne, P Jänis, C Ribeiro, K Hugl, Device-to-Device communications; functional prospects for LTE-Advanced networks, in Proceedings of the International Conference on Communication (Dresden, 14-18 June 2009), pp. 1-6

3. P Jänis, CH Yu, K Doppler, C Ribeiro, C Wijting, K Hugl, O Tirkkonen, V Koivunen, Device-to-Device communication underlaying cellular communications systems, Int. J. Commun. Netw. Syst. Sci. 2(3) (2009)

4. T Nakamura, S Nagata, A Benjebbour, Y Kishiyama, T Hai, S Xiaodong, Y Ning, L Nan, Trends in small cell enhancements in LTE advanced. IEEE Commun. Mag. 51(2), 98-105 (2013)

5. L Lei, Z Zhong, C Lin, X Shen, Operator controlled device-to-device communications in LTE-advanced networks. IEEE Wireless Commun 19(3), 96-104 (2012)

6. S Hakola, T Chen, J Lehtomaki, T Koskela, Device-to-device (D2D) communication in cellular network-performance analysis of optimum and practical communication mode selection, in Proceedings of the International Wireless Communication and Networking Conference (Sydney, 18-21 April 2010), pp. 1-6

7. H Min, J Lee, S Park, D Hong, Capacity enhancement using an interference limited area for device-to-device uplink underlaying cellular networks. IEEE Trans. Wireless Commun. 10(12), 3995-4000 (2011)

8. P Jänis, V Koivunen, C Ribeiro, J Korhonen, K Doppler, K Hugl, Interference-aware resource allocation for Device-to-Device radio underlaying cellular networks, in Proc. of IEEE Vehicular Technology Conference, Spring (Barcelona, 26-29 April 2009), pp. 1-5
9. CH Yu, O Tirkkonen, K Doppler, C Ribeiro, Power optimization of device-to-device communication underlaying cellular communication, in Proceedings of the International Conference on Communication (Dresden, 14-18 June 2009), pp. 1-5

10. K Doppler, CH Yu, C Ribeiro, P Janis, Mode Selection for device-to-device communication underlaying an LTE-advanced network, in Proceedings of the International Wireless Communications and Networking Conference (Sydney, 18-21 April 2010), pp. 1-6

11. M Jung, K Hwang, S Choi, Joint mode selection and power allocation scheme for power-efficient device-to-device (D2D) communication, in Proceedings of the IEEE Vehicular Technology Conference (Yokohama, 6-9 May 2012), pp. 1-5

12. C Zhong, S Jin, KK Wong, Dual-hop systems with noisy relay and interference-limited destination. IEEE Trans. Commun. 58(3), 764-768 (2010)

13. T Cover, AE Gamal, Capacity theorems for the relay channel. IEEE Trans Inf. Theory. 25(5), 572-584 (1979)

14. B Rankov, A Wittneben, Spectral efficient protocols for half-duplex fading relay channels. IEEE J. Sel. Areas in Commun. 25(2)، 379-389 (2007)

15. MN Khormuji, EG Larsson: Cooperative transmission based on decode-and-forward relaying with partial repetition coding. IEEE Trans. Wireless Commun. 8(4), 1716-1725 (2009)

16. $\mathrm{CH}$ Yu, K Doppler, C Ribeiro, O Tirkkonen, Resource sharing optimization for device-to-device communication underlaying cellular networks. IEEE Trans. Wireless Commun. 10(8), 2752-2763 (2011)

17. Y Ni, S Jin, R Tian, KK Wong, H Zhu, S Shao, Outage analysis for device-to-device communication assisted by two-way decode-and-forward relaying, in Proceeding of the International Conference on Wireless Communications and Signal Processing (Hangzhou, 24-26 Oct 2013), pp. 1-6

18. LK Saliya Jayasinghe, P Jayasinghe, N Rajatheva, M Latva-aho, MIMO physical layer network coding based underlay device-to-device communication, in Proceedings of the International Symposium on Personal Indoor and Mobile Radio Communications (London, 8-11 Sept 2013), pp. 89-94

19. C Ma, G Sun, X Tian, K Ying, Y Hui, XB Wang, Cooperative relaying schemes for device-to- device communication underlaying cellular networks, in Proceedings of the IEEE Global Communications Conference (Altlanta, 9-13 Dec 2013), pp. 3890-3895

20. DH Lee, S Kim, J Lee, J Heo, Performance of multihop decode-and-forward relaying assisted device-to-device communication underlaying cellular networks, in Proceedings of the International Symposium on Information Theory and its Applications (Honolulu, 28-31 Oct 2012), pp. 455-459

21. S Wen, X Zhu, Y Lin, Z Lin, X Zhang, DC Yang, Achievable transmission capacity of relay-assisted device-to-device (D2D) communication underlay cellular networks, in Proceedings of the IEEE Vehicular Technology Conference (Las Vegas, 2-5 Sept 2013), pp. 1-5

22. LF Wang, T Peng, Y Yang, WB Wang, Interference constrained D2D communication with relay under laying cellular networks, in Proceedings of the Vehicular Technology Conference (Las Vegas, 2-5 Sept 2013), pp. 1-5

23. LF Wang, T Peng, Y Yang, WB Wang, Interference constrained relay selection of D2D communication for relay purpose underlaying cellular networks, in Proceedings of the Wireless Communications, Networking and Mobile Computing (Shanghai, 21-23 Sept 2012), pp. 1-5

24. M Kountouris, D Gesbert, T Salzer, Distributed transmit mode selection for MISO broadcast channels with limited feedback: switching from SDMA to TDMA, in Proceedings of the Workshop on Signal Processing Advances in Wireless Communications (Recife, 6-9 July 2008), pp. 371-375

25. J Zhang, RW Heath, M Kountouris, JG Andrews, Mode switching for the multi-antenna broadcast channel based on delay and channel quantization. EURASIP J. Adv. Signal Process. 2009, 1 (2009)

26. H Min, W Seo, J Lee, S Park, D Hong, Reliability improvement using receive mode selection in the device-to-device uplink period underlaying cellular networks. IEEE Trans. Wireless Commun. 10(2), 413-418 (2011)

doi:10.1186/1687-6180-2014-138

Cite this article as: Ni et al:: Transmission mode switching for device-to-device communication aided by relay node. EURASIP Journal on Advances in Signal Processing 2014 2014:138. 